\title{
Range image registration for industrial inspection
}

\author{
Carles Matabosch ${ }^{a}$, Joaquim Salvi ${ }^{a}$, David Fofi ${ }^{b}$ and Fabrice Meriaudeau ${ }^{b}$ \\ ${ }^{a}$ University of Girona,Institut d'Informatica i Aplicacions, Campus Montilivi, 17017, Girona, \\ Spain; \\ ${ }^{b}$ Laboratoire Electronique, Informatique et Image, University of Burgundy, rue de la fonderie \\ 12, 71200, Le Creusot, France
}

\begin{abstract}
Building of three-dimensional models is an important topic in computer vision. Range finders only let to reconstruct a partial view of the object. However, in most part of applications a full reconstruction is required. Many authors have proposed several techniques to register 3D surfaces from multiple views. In this paper, a survey of the most common techniques is presented. Furthermore experimental results are performed, and a 3D model is obtained.
\end{abstract}

Keywords: Inspection, Modelling, Range Image, Registration

\section{INTRODUCTION}

Reconstruction of 3D objects is an important topic in computer vision with many applications, such as reverse engineering, robot navigation, mould fabrication and visual inspection, among others. However, most recent methods to get 3D models may only reconstruct a part of the object from a mechanical scanning. In order to get a complete model, multiple range images of the same object must be merged. Range image, also called $21 /{ }_{2} \mathrm{D}$ image, is formed by a common 2D image with some additional information which leads to compute directly the $3 \mathrm{D}$ surface. A $2^{1 / 2} \mathrm{D}$ image is given by laser scanning, ${ }^{1}$ pattern projection ${ }^{2}$ or stereovision. ${ }^{3}$ In order to register multiple images, Euclidean motion between views must be determined. Some authors supposed that initial approximation of this motion is given. In that situation, the problem is solved by minimizing the distance between consecutive range images, with the aim of obtaining a Fine Registration. However, in most applications the initial motion is not available. In this case, a Coarse Registration must be previously solved in order to compute an initial estimation of the motion between views.

This paper presents a survey on range image registration. First, a classification on 3D image registration is presented in section 2. Then, section 3 and 4 detail the methods based on Coarse registration and Fine registration, respectively. In section 5 a proposal to fusion more than two views is presented. The experimental results focused on both registration techniques are presented in section 6 . The article ends with conclusions.

\section{CLASSIFICACION}

Registration techniques are based on finding the Euclidean motion between some range images. The presented techniques differ whether initial information is required, so that only a Coarse registration can be obtained without an initial guess. Besides, if an estimated motion between views is available, a Fine registration is computed. The classification of the surveyed methods is shown in table 1.

In Coarse registration, the main goal is to determine an initial estimation of the rigid motion between two clouds of 3D points by determining correspondences. Although there are other methods, most important can be classified as: a) Point Signature, b) Spin Image, c) Principal Component Analysis and d) Genetic Algorithms. All of them are presented in section 3 .

Further author information: (Send correspondence to C.M.)

C.M.: E-mail: cmatabos@eia.udg.es, Telephone: 0034972419812

J.S.: E-mail: jsalvi@eia.udg.es, Telephone: 0034972419812

D.F.: E-mail: d.fofi@iutlecreusot.u-bourgogne.fr, Telephone: +33 (0)385731126

F.M.: E-mail: fabrice@iutlecreusot.u-bourgogne.fr, Telephone: +33 (0)385731077 
Table 1. Classification of Registration methods

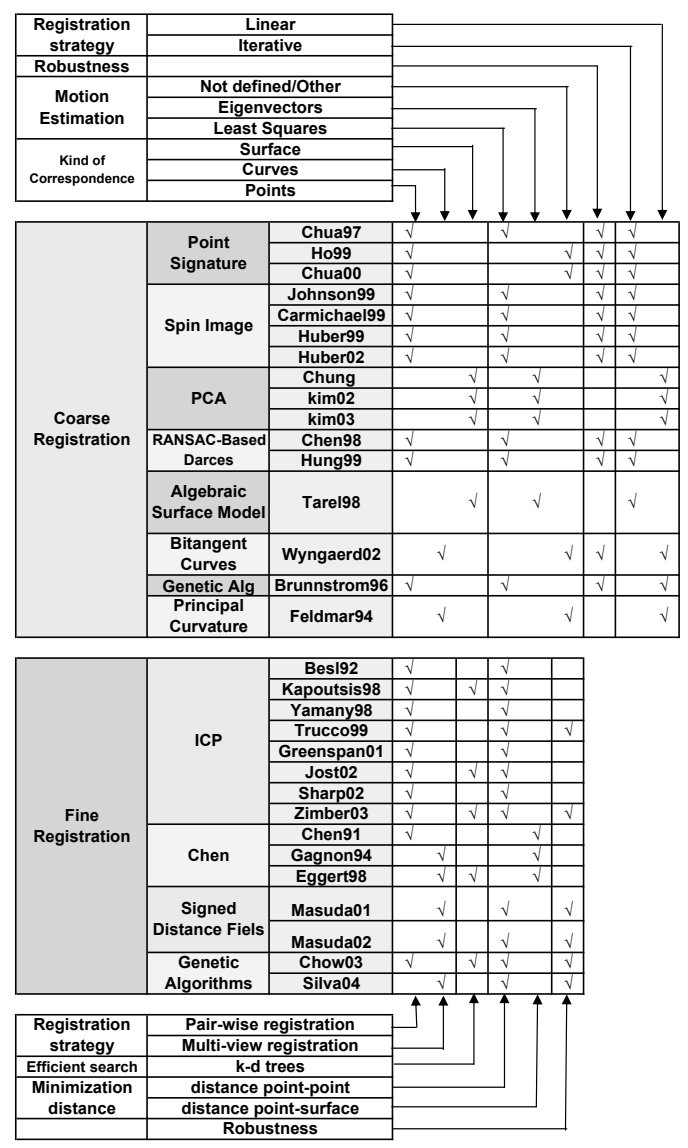

Table 1 also classifies the methods in terms of: a)kind of correspondence; b)Algorithm used to estimate the motion; c)Robustness of the method and d) Registration strategy.

In Fine registration, the goal is to get a better solution by minimizing an initial guess provided by Coarse registration or by a mechanical system. Basically, these methods can be classified in: a) Iterative Closest Points, b) Method of Chen, c) Signed Distance Fields and d) Genetic Algorithms. All of them are presented in section 4.

Table 1 also classifies the coarse registration methods in terms of: a)the registration strategy, i.e. Pair-wise or multi-view registration depending on the number of views that are aligned in every iteration; b)the used of an efficient search such as k-d trees in order to speed up the algorithm; c)the way of computing the minimization distances whether they use distances point-to-point or point-to-plane; and d) the way of computing the initial motion.

\section{COARSE REGISTRATION}

Coarse registration methods search for an initial estimate of the motion between pairs of consecutive 3D views leading to the complete registration of the surface. In order to compute this motion, distances between correspondences in different views are minimized. Most common correspondences are points, curves or surfaces. The main problem of this sort of registration is the high computation cost in finding correspondences, and particularly when features are extracted to match them. In this section, most important techniques are explained. 


\subsection{Point Signature}

Point correspondences are sought prior to finding the Euclidean motion. In order to establish these correspondences, a point representation called Point Signature is computed. This is based on the information of the neighbor points. This method characterizes some points of the first cloud, and then looks for possible correspondences for each point in the second cloud.

Point signature was introduced by Chua. ${ }^{4}$ For a given point $p$, the curve of the surface that intersects with a sphere of radius $r$ centered to $p$ gives the contour of points $(\mathrm{C})$. They are represented in a new coordinate frame centered at $p$. The orientation axes are given by the normal vector $\left(n_{1}\right)$ at $p$, a reference vector $\left(n_{2}\right)$ and the vector obtained by their cross-product. All points on $\mathrm{C}$ are projected to the tangent plane giving a curve $\mathrm{C}$ '. The vector $n_{2}$ is computed as the unit vector from $p$ to a point on $\mathrm{C}^{\prime}$ which gives the largest distance. Then, every point on $\mathrm{C}$ can be characterized by a) the signed distance between its own correspondent in $\mathrm{C}^{\prime}$ and b) a clockwise rotation angle $\theta$ from the reference vector $n_{2}$. Depending on the resolution, different $\Delta \theta^{\prime}$ 's are chosen. Then, the point signature can be expressed as a set of distances in each $\theta$ from $0^{\circ}$ to $360^{\circ}$. Finally point signatures from 2 views are compared allowing for an error tolerance. The matching process is very fast and efficient. However, the method requires a lot of time for computing point signatures, especially if the surface is expressed in clouded points, because it is necessary to search for the neighbors and interpolate the surface to determine the region that intercepts with the circle.

The principal problem of this method is that it is necessary to identify the neighbors to construct the pointsignature. Furthermore, if the surface to register are clouds of points, it is necessary to interpolate the surface in order to find points belonging to the surface of the sphere or use the nearest point to the surface, which introduces error in the method.

\subsection{Spin Image}

Spin image ${ }^{5}$ is a $2 \mathrm{D}$ image that characterizing a point using the information of the surface near to it. From a given point, a normal vector is computed approximating the points of the local surface with a plane. Then two distances are computed to determine the spin image: a) distance $\alpha$ between the normal vector to a point in the range image and b) distance $\beta$ between the tangent plane to a point.

$$
\begin{gathered}
\alpha=\sqrt{\|x-p\|^{2}-(n(x-p))^{2}} \\
\beta=n(x-p)
\end{gathered}
$$

where $p$ is the $3 \mathrm{D}$ point, $n$ the normal vector at the point where the spin image is computed, and $x$ is the set of neighbor points used to generate the spin image. Using these distances, a table is constructed, representing $\alpha$ along the $\mathrm{x}$-axis and $\beta$ along the $\mathrm{y}$-axis. Each cell of this table contains the number of points that belong in this region. In order to choose the number of the bins (elements of the table), which determines the resolution of the image, the double length of the triangle mesh is selected.

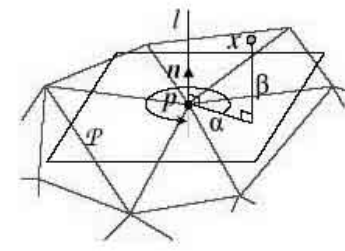

(a)

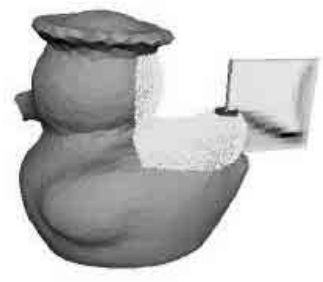

(b)

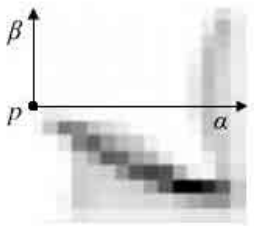

(c)

Figure 1. Example of spin image construction. a) Representation of the axis of the spin image . b) Rotating the plane respect to the normal vector, the spin image is obtained and c) Final result of the spin image 
Some spin images are computed in the first view and then for each one, the best correspondences is searched for in the second view. When point correspondences are found, outliers are eliminated using the mean and the variance of the errors. The rigid transformation is computed using the best correspondences founded. The Spin Image is similar to Point Signature, however the characterization of points is easier in Spin Images and more accurate. Furthermore, a surface is not required, the range image can be characterized with a cloud of points. The main problem of this method is the variation of the spin image respects to the resolution of the method. To solve this problem, Carmichael ${ }^{6}$ used the face-based spin image. Interpolated points inside the triangular mesh are used to equalize the points of the surface. Other approaches are presented to solve shadow problems. ${ }^{7}$

\subsection{Principal Component Analysis}

This method is based on using the main axis direction to align partial overlapping range images. If overlapping region is important, both main axis are almost coaxial. Therefore, the transformation matrix can be found as one that aligns both axes only by applying a simple multiplication (see eq. 5). This method is very fast respects with others that identify point or curve correspondences. However, it is necessary that the overlapping region is very significant part of the image in order to obtain good results.

Chung proposed this new registration algorithm using the direction vectors of clouded points. ${ }^{8}$ The method involves calculating the covariance matrix of each range image:

$$
\operatorname{Cov}=\frac{1}{N} \sum_{i=0}^{N-1}\left(p_{i}-\bar{p}\right)\left(p_{i}-\bar{p}\right)^{T}
$$

where $\bar{p}$ is the center of mass of the clouded points. Then, it is possible to compute the direction $U_{i}$ of the main axis by singular value decomposition:

$$
\operatorname{Cov}_{i}=U_{i} D_{i} U_{i}^{T}
$$

The rotation is determined by the product of the eigenvector matrices:

$$
R=U_{1} U_{2}^{-1}
$$

and the translation by the difference between centers of mass of both clouded points expressed with respect to the same axis:

$$
t=\bar{\mu}_{2}-R \bar{\mu}_{1}
$$

Principal component analysis is very fast, however it can be used effectively when there is a sufficient number of points. It can be used with few points ( 200 points) with good results, but only when the overlapping region is a significant percentage of the range image. This method attains an accurate solution when most part of points are common. Results are less accurate when the overlapping region constitutes a smaller part of the image, for example only about $50 \%$, but may be good enough to apply a subsequent fine registration method. The main problem of Principal Component Analysis is its limitation to cope with surfaces that contain symmetries. In this way, if the eigenvalues obtained representing two axis are similar, the order of these axis can change in the matrix $U_{i}$, and the final result obtained is completely different from the right solution.

\subsection{RANSAC-Based Darces}

RANSAC-Based method ${ }^{9}$ is based on finding the best three point correspondences between two range images in order to obtain an estimation of the Euclidean motion. Three points are the minimum required to compute the motion between both surfaces.

Three points (primary, secondary and auxiliary) of the first cloud are characterized by the distance between them. Then, each point in the second cloud is hypothesized as the primary point. Next, the secondary point is searched for from the points located at the same distance as between the primary and secondary points in the first cloud. If no point is found, another primary point is tested. Otherwise, a point in the second cloud that satisfies the distances defined in the triplet of the first cloud is found. Once a triplet is identified that matches 
the first, it is then possible to determine the rigid transformation. This search is repeated for several three points in the first image in order to obtain different possible Euclidean motions. The final transformation is the one with the greatest number of overlapping points in two views.

The results of this method are very good, because of robustness to outliers, as a result of searching for the best possible matching triplet. Theoretically, the precision of the results increase with the resolution of the image, however computing time is its principal problem, especially when lots of points are used.

\subsection{Genetic Algorithm}

Genetic algorithm (GA) is an evolutionary technique which generates possible solutions in a maximization problem. Each possible solution is known as "chromosome" or "genome". Chromosomes are combined or mutated to breed new individuals. Crossover is the kind of recombination of chromosomes found in sexual reproduction in nature, is often also used in GAs. Here, an offspring's chromosome is created by joining segments chosen alternately from each of two parents' chromosomes which are of fixed length.

Brunnstrm used a genetic algorithm to find point correspondences between range images. ${ }^{10}$ The main goal of this method is to find a vector which contains the $n$ indices of correspondences between both range images, where $n$ is the number of points in the second cloud of points. In these algorithms, it is necessary to define a fitness function which measures the quality of each possible solution (the vector of point correspondences).

To determine this function, four invariants between two pairs of correspondences are used:

$$
\begin{gathered}
\left\|\vec{v}_{i j}\right\|=\left\|\vec{r}_{j}-\vec{r}_{i}\right\| \\
\cos \left(\theta_{i j}\right)=\frac{\vec{n}_{j} \cdot \vec{v}_{i j}}{\left\|\vec{n}_{j}\right\| \cdot\left\|\vec{v}_{i j}\right\|} \\
\cos \left(\theta_{j i}\right)=\frac{\vec{n}_{i} \cdot \vec{v}_{j i}}{\left\|\vec{n}_{i}\right\| \cdot\left\|\vec{v}_{j i}\right\|} \\
\cos \left(\beta_{j i}\right)=\frac{\left(\vec{n}_{j} \times \vec{v}_{i j}\right) \cdot\left(\vec{n}_{i} \times \vec{v}_{i j}\right)}{\left\|\vec{n}_{i}\right\| \cdot\left\|\vec{n}_{j}\right\| \cdot\left\|\vec{v}_{i j}\right\|^{2}}
\end{gathered}
$$

Using these invariants, the quality of these correspondences are computed by analyzing the error of the distance and the error in the normal parameters as follows:

$$
\begin{aligned}
& q_{d}\left(\alpha_{i}, \alpha_{j}\right)=e^{-\frac{\left[\left\|\vec{v}_{\alpha_{i} \alpha_{j}\|-\|} \vec{v}_{i j}\right\|\right]^{2}}{2 \sigma^{2}}}
\end{aligned}
$$

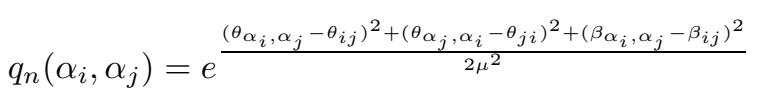

$$
\begin{aligned}
& Q\left(\alpha_{i}, \alpha_{j}\right)=q_{d}\left(\alpha_{i}, \alpha_{j}\right) q_{n}\left(\alpha_{i}, \alpha_{j}\right)
\end{aligned}
$$

where $\sigma$ and $\mu$ are experimental parameters that must be estimated, and parameters $k_{i j}$ are the parameters of the second range image, and $k_{\alpha_{i}, \alpha_{j}}$ represent the parameters of the possible correspondences in the first range image. Then, the quality of a correspondence $\alpha_{i}$ can be computed as the sum of the quality of all pair of correspondences between $\alpha_{i}$ and all points.

$$
Q\left(\alpha_{i}\right)=\sum_{j \neq 1} Q\left(\alpha_{i}, \alpha_{j}\right)
$$

However, this measure represents the quality of one pair of correspondences, and the fitness function expresses the global matching quality, which is expressed as a function of $Q\left(\alpha_{i}\right)$.

$$
f(\vec{\alpha})=e^{\sqrt{\sum_{i} Q\left(\alpha_{i}\right)}}
$$


When the fitness function is defined, the cross-over and mutation probabilities must be fixed to characterize the algorithm. In the problem of searching for correspondences, the mutation is not very important, because near indices do not imply near points in the space. Therefore, the author fixed the probability of mutation to $1 \%$, and a crossover of $90 \%$ as the parameters of the Genetic Algorithm.

Finally, a stopping criterion is defined, a problem not very studied in genetic algorithms. Three different approaches were presented: a) Fixing a \% of good correspondences (computing by finding closest points); b) a certain number of iterations before the fitness function does not increase and c) after a certain number of iterations.

When the algorithm finalizes, the Euclidean motion can be computed because the chromosome that gives the greatest value in the fitness function contains the points correspondences, however, not all correspondences are good, and bad correspondences must be rejected to compute a good Euclidean motion. To do this, only the $30 \%$ of correspondences with greatest values in the parameter $Q\left(\alpha_{i}\right)$ are used to compute the motion by means of SVD method. The main problem of this algorithm is the high computational time to find a good solution.

All Coarse Registration methods gives us a estimation of the motion between two range images although no information of the orientation is available. However, in normal situations, the quality of their results are not enough to correctly register range images. To solve this problem, Fine registration methods are used to find a better solution.

\section{FINE REGISTRATION}

The term Fine registration is used when an initial estimation of the motion is previously known and the main goal is to converge to a more accurate solution. In order to solve this problem, a distance function is minimized. Some authors used the distance between point correspondences while others used the distance point-to-plane. During the last years, some methods have been presented. Most relevant are commented in this section.

\subsection{Iterative Closest Point (ICP)}

Iterative Closest Point is a Pair-wise registration algorithm presented by Besl. ${ }^{11}$ The goal of this method is to obtain an accurate solution by minimizing the distance between point correspondences, known as closest point. Using an initial estimation between two range images, all points are transformed into a common reference frame applying this Euclidean motion. Then, for each point of one image, the Closest Point is searched in the second image. The goal of this method is to minimize the distance between these correspondences:

$$
f=\frac{1}{N_{p}} \sum_{i=1}^{N_{p}}\left\|\vec{m}_{i}-R\left(\vec{q}_{R}\right) \vec{p}_{i}-\vec{t}\right\|^{2}
$$

where $\mathrm{R}$ is the estimated rotation matrix between both views, $\vec{t}$ the translation vector between them, and $p_{i}$ and $m_{i}$ are points from both range images. When motion parameters are found, this new motion is applied to the first surface and repeated the process until the improvement in the error is smaller than a threshold.

This method obtains good results and it is robust to noise, its drawbacks are: a) the presence of nonoverlapping regions which is one of the causes of bad alignments and b)non-convergence problem due to bad initial estimation of the motion.

To solve this problem, several authors presented improvements of this technique. Trucco ${ }^{12}$ implemented the RICP method, a robust algorithm based on the use of Least Median of Squares. The method is based on registering range images with a set of points $(m)$, and computing with different sets the sufficient number of time with the aim of finding a combination without outliers. Monte Carlo algorithm is used in order to estimate the number of required combinations. In each combination, the rotation matrix is estimated by least-squares. When all combinations are computed, the solution that minimizes the median of the residuals is chosen. Finally, all correspondences with a residual greater than $2.5 \sigma$ are removed and rotation is computed from the rest of point pairs. A robust standard deviation ${ }^{13}$ is used to estimate $\sigma$. Translation can be computed by the subtraction of the center of mass of both corresponding points expressed in the same coordinate frame. 
Zim $\beta \mathrm{er}^{14}$ proposed another robust method based on the outlier thresholding known as the Picky ICP algorithm. Different levels of resolution (similar to $\mathrm{Jost}^{15}$ ) and the nearest neighbor algorithm are used to reduce the computational time. However, the main advantage is that at each iteration only the point pairs with smallest distance among them are used to compute the motion. In order to select the best points to compute the motion, the standard deviation is estimated and correspondences with distances smaller than a chosen multiple of this estimated deviation are selected as the best correspondences.

\subsection{Method of Chen}

Chen ${ }^{16}$ proposed another Pair-wise Registration algorithm, based on minimizing the distance between points and planes. Distances between points on the first image and tangent planes in the second one are minimized. Given a point in the first image, the intersection between normal vector to this point to the second surface are searched for. At the intersection point, the tangent plane is computed, and the distance between this plane and the given point is the distance to minimize.

An algorithm to find the intersection between lines and digital surfaces is presented, because the intersection of a line in a cloud of points requires a high computational time.

When the distances are found, the transformation that minimizes these distances is found by least squares, and the process is repeated until the change in the distances between points and planes are less than a threshold.

An improvement of this method was presented by Gagnon ${ }^{17}$ to speed up the search for the distance between points and planes and obtain it with more accuracy. First of all, the surface of the second range image is projected on a plane partitioned in a set of squares like a chessboard. The normal vector at a given point in the first range image is projected on such a partitioned plane obtaining two intersection points at the borders of every square. The square where the sign in the z-coordinate of both points changes is selected as the intersection point. Then, the plane tangent to such correspondence point is computed and the distance to the given point in the first range image evaluated.

The method of Chen theoretically requires less iterations than ICP algorithm. However, in practice, the convergence to the correct solution is not ensure. Furthermore, the time required to find the distance point-toplane is considerably higher with respect to the distance point-to-point.

\subsection{Matching Signed Distance Fields}

Masuda ${ }^{18}$ presented a registration algorithm based on Matching Signed Distance Fields. The author presented a method that registers and integers all range images and rejects outliers at the same time.

All views are transformed into a common reference frame using the parameters of the estimated motion. Using this integrated model, the closest points in each key point are searched in order to establish correspondences. The key points are points located on a 3D grid of fixed interval $\delta$. Furthermore, the distance of searching for the closest point is limited to $\sqrt{3} \delta$.

The process is composed by the following steps:

1. Initialization: Determining the initial values of the motion parameters, $T_{0}^{\alpha}$

2. Determination of the closest point $p_{i}^{\alpha}$ for each key point $p$.

3. Determine the new motion parameters $T_{i+1}^{\alpha}$ using the correspondences between $p$ and $p_{i}^{\alpha}$

Steps 2 and 3 are repeated until the residual error converges. When this process is ended, the integration is ended too, because the set of control points $p$ used to register the range views are the points of the integrated model. Furthermore, normals of these points are found interpolating the normals of the different closest points for each key point.

The advantage of this method is that it registers all views at the same time, which implies a more robust solution and avoid the error propagation problem of pair-wise registration methods. Furthermore, the final result of the algorithm is an integrated model, because there are no redundant data in the overlapping regions. On the other hand, the multi-view strategy is not possible in several applications, when the motion between two pairs of range views before acquiring the following view is required. 


\subsection{Genetic Algorithms}

A dynamic genetic algorithm to solve the registration problem is presented by Chow. ${ }^{19}$ The goal of this method is to find a chromosome composed by the 6 parameters of the motion that aligns accurately a pair of range images. The chromosome is composed by the three components of the translation vector $\left(T_{x}, T_{y}\right.$ and $\left.T_{z}\right)$ and the three angles of rotation along each axis $(\alpha, \beta$ and $\theta)$.

Then, the registration error is minimized. To do this, the median of the registration error is chosen as a fitness function. The median is a statistic more robust than the mean.

$$
F(T)=\operatorname{Median}\left(E_{i}\right), \text { where } E_{i}=\min \left|T\left(p_{i}\right)-m_{j}\right|
$$

Therefore, only a sub-sample group of points of the first image are analyzed to find the error, in order to decrease the computational time.

With the aim of minimizing the fitness function, the cross-over and the mutation operations have to be defined. The cross-over operation consists of the combination of some genes of two chromosomes in order to create a new one. The author randomly selected the number of genes to be swapped, and then he selected the genes to be swapped. The cross-over operation is good when the solution is far from the actual chromosome, therefore, it is not very interesting to obtain a better solution when it is close to the correct one. To solve this problem, the mutation operation is applied.

In the mutation, one gene is randomly selected and a value $[-M V,+M V]$ is added to the initial value of the gene, and a new chromosome is obtained. However, the choice of the optimal value of the MV is not easy because when the solution is not very good, a larger value of MV is needed, however, close to the correct solution, the value MV has to be smaller. To solve this problem a dynamic value is chosen.

Additionally, when the GA leads to a good solution, another genetic algorithm with a smaller value of the boundary is applied until no improvements are obtained with the new boundary. This method is very robust and accurate, however the computing time is an important drawback.

\section{A NEW ALGORITHM TO FUSION ALL VIEWS}

In this section, we present a technique to minimize the global registration error detecting the best path to transform all views to the reference frame. The proposal is to compute the transformation of each image with the reference frame throughout the path with minimal residual error. This error is computed as the mean of the distances between point correspondences of both range images for every Pair-wise registration.

As the connections between views are unknown, all views are registered, obtaining a transformation matrix between all of them. We obtain a connectivity graph between all views, where the cost of the path between views $i$ and $j$ is the registration error obtained in the registration. In this situation, we obtained a full connectivity graph, where each view can be transformed to the reference one (first view) using different paths between views. In order to obtain the best possible model, we have to determine the path that transforms all views to the reference frame related to the minimum cost. Dijkstra algorithm is applied to determine optimal path in graphs to solve this problem, obtaining a reduced graph.

The final transformation between each view and the reference frame is the product of all transformations matrix in the optimal path, the global registration error related to the same view is the sum of the partial error registrations involved in the path.

When all global errors are obtained, all views whose global error are smaller than $2 \sigma$ are fused, creating an integrated model, where $\sigma$ is the variance of the global errors. Using this variance, bad registered views are not integrated to the model. However, the final model can be incomplete due to the non included views. Generally, bad alignments in the registration process are caused by a little overlapping region. To solve this problem, all not included views are registered respect to the integrated model. The transformation matrix obtained in this situation is directly the matrix that transforms all points to the reference frame.

In order to quantify the errors of our proposal, and to compare with Pair-wise traditional registration, the error of registration is computed for each view. Theoretically, the identity matrix is the solution for each 
Table 2. Registration errors in Rotation

\begin{tabular}{|c|c|c|c|}
\hline & \multicolumn{3}{|c|}{ Rotation angle error } \\
\hline View & Pair-Wise & Without Correction & Our Proposal \\
\hline 2 & $0.32^{\circ}$ & $0.32^{\circ}$ & $0.32^{\circ}$ \\
\hline 3 & $0.71^{\circ}$ & $1.90^{\circ}$ & $1.90^{\circ}$ \\
\hline 4 & $6.40^{\circ}$ & $3.49^{\circ}$ & $3.49^{\circ}$ \\
\hline 5 & $8.23^{\circ}$ & $1.29^{\circ}$ & $1.29^{\circ}$ \\
\hline 6 & $8.34^{\circ}$ & $1.20^{\circ}$ & $1.20^{\circ}$ \\
\hline 7 & $9.11^{\circ}$ & $0.57^{\circ}$ & $0.57^{\circ}$ \\
\hline 8 & $9.75^{\circ}$ & $0.49^{\circ}$ & $0.49^{\circ}$ \\
\hline 9 & $13.87^{\circ}$ & $11.15^{\circ}$ & $0.31^{\circ}$ \\
\hline 10 & $17.1^{\circ}$ & $16.42^{\circ}$ & $0.32^{\circ}$ \\
\hline
\end{tabular}

transformation matrix. The difference between the real matrix and the identity one is the error of the registration. In order to quantify this error, the rotation matrix is expressed in axis-angle representation. The value of this angle is a measure of the quality of the registration. Null angle is the ideal value for a correct registration. Three algorithms are compared, the first one is the traditional Pair-wise registration, where only consecutive views are registered. The second one is our proposal without the elimination of bad registered views. The final algorithm is our proposal with the correction of bad aligned views (see table 2). The results show that the first algorithm is very sensitive to error propagation. The second one minimizes the errors in the registration path and implies a minimization in the registration errors, however when two views are bad registered the error is considerable. The final algorithm presents good results for all views.

\section{EXPERIMENTAL RESULTS}

In the last sections, different registration methods are explained, and their advantages and drawbacks are commented. However, the best way to compare them is to evaluate their results for identical conditions. In this chapter, most representative methods are compared using synthetic and real data. So as to compare the different methods, different experiments are performed in order to evaluate the errors of methods in the same conditions. To do this, different types of errors are computed, and this information helps us to decide which method is the best for each situation. The computing time is also provided for each method.

The best way for comparing different methods is to perform simulations using synthetic data, for which correct value of the result is known, enabling an easy comparison of the different methods. The parameters computed are the error in Rotation, the error in Translation and 3D error.

In order to evaluate errors in rotation, we express it in axis-angle representation. This representation is based on an axis direction and an angle of rotation around this vector. When rotation matrix between both range images and the synthetic rotation matrix (known in synthetic data because the motion can be controlled by the user) are expressed in axis-angle, errors is computed as follows: first of all, the angle between both axes $(\gamma)$ are computed as an error, and then the difference between both angles of rotation $(\alpha, \beta)$ are computed as a second error.

With the aim of computing the error of translation, that expresses the difference distance between origin of system coordinate in synthetic case and with the one obtained by the registration method, the norm of the difference between both translation vectors is computed.

The 3D error states the mean of the distance between point correspondences when all images are expressed in the same coordinate system. To compute this, first of all both range images must be represented in the same coordinates system applying the Euclidean motion computed previously, and then the norm of all distances between point correspondences are computed. This error is less accurate than the other presented before, however it is very important for unknown real motion where it is impossible to determine the errors of the motion. Experimental results are shown in tables 3 and 4 . The final results of the registration are shown in figure 2 . 
Table 3. Experimental results of two real range images using Coarse registration methods

\begin{tabular}{|c|c|c|c|c|c|}
\hline Points & Method & Translation error $(\mathrm{mm})$ & $\alpha$ - $\beta(\mathrm{rad})$ & $\gamma$ & time $(\mathrm{s})$ \\
\hline \multirow{6}{*}{100} & Ransac-Based & 28.55 & 0.09 & 1.70 & 19.14 \\
& PCA & 69.42 & 1.93 & 1.31 & 0.28 \\
& SpinImage & 55.84 & 0.90 & 1.75 & 125.48 \\
& GenAlg & 52.89 & 0.96 & 1.19 & 3381.7 \\
\hline \multirow{6}{*}{250} & Ransac-Based & 26.92 & -0.25 & 1.27 & 530.44 \\
& PCA & 53.12 & 1.06 & 1.47 & 0.59 \\
& SpinImage & 54.08 & 1.29 & 1.02 & 245.56 \\
& GenAlg & $\infty$ & $\infty$ & $\infty$ & $\infty$ \\
\hline \multirow{6}{*}{500} & Ransac-Based & $\infty$ & $\infty$ & $\infty$ & $\infty$ \\
& PCA & 69.89 & 1.92 & 1.33 & 6.44 \\
& SpinImage & 69.36 & 2.14 & 1.73 & 1183.90 \\
& GenAlg & $\infty$ & $\infty$ & $\infty$ & $\infty$ \\
\hline
\end{tabular}

Table 4. Experimental results of Fine registration methods with real data

\begin{tabular}{|c|c|c|c|c|c|}
\hline Points & Method & Translation error $(\mathrm{mm})$ & $\alpha-\beta(\mathrm{rad})$ & $3 \mathrm{D}$ error $(\mathrm{mm})$ & time $(\mathrm{s})$ \\
\hline \multirow{6}{*}{500} & Besl & 3.34 & -0.09 & 4.18 & 1.41 \\
\hline & $\operatorname{Zim} \beta$ er & 0.68 & -0.06 & 2.35 & 1.95 \\
\hline & Jost & 2.01 & -0.07 & 4.54 & 0.36 \\
\hline & Trucco & 2.87 & -0.05 & 3.93 & 19.33 \\
\hline & Gagnon & 9.14 & -0.11 & 26.665 & 0.75 \\
\hline & Chow & 0.27 & 0.0 & 4.60 & 154.05 \\
\hline \multirow{6}{*}{1000} & Besl & 3.47 & -0.09 & 3.78 & 5.22 \\
\hline & $\operatorname{Zim} \beta$ er & 0.47 & -0.02 & 1.42 & 7.97 \\
\hline & Jost & 2.38 & -0.08 & 3.70 & 1.38 \\
\hline & Trucco & 2.71 & -0.05 & 5.13 & 33.95 \\
\hline & Gagnon & 23.44 & -0.57 & 22.36 & 2.39 \\
\hline & Chow & 0.12 & -0.01 & 4.51 & 281.61 \\
\hline \multirow{6}{*}{5000} & Besl & 3.12 & -0.08 & 3.20 & 57.36 \\
\hline & $\operatorname{Zim} \beta$ er & 0.26 & -0.02 & 0.85 & 281.58 \\
\hline & Jost & 2.24 & -0.09 & 2.83 & 9.14 \\
\hline & Trucco & 2.57 & -0.05 & 1.77 & 149.59 \\
\hline & Gagnon & 30.40 & -0.24 & 24.86 & 8.78 \\
\hline & Chow & 0.06 & 0.0 & 3.57 & 1776.00 \\
\hline
\end{tabular}

Furthermore complete reconstruction can be obtained by Pair-wise registration if our approach is used as presented in section 5. The results of this method are shown in figure 3 , where they are compared with the traditional registration methods.

\section{CONCLUSIONS}

This work surveys the most common registration methods. These kind of methods are used to build complete models of real objects. The main classification is based on the accuracy of the results. Coarse registration techniques are used when an initial estimation of the Euclidean motion is unknown. However, results obtained with these techniques present a rough accuracy. The procedure of these techniques is based on finding correspondences between clouds of points and then computing the Euclidean motion. Different kind of correspondences can be used, as points, curves, surfaces and directional vectors. Fine registration methods are based on converging to a solution from an initial estimation of the rigid motion. Depending on the method used, a better initial guess is required because some methods have problems of convergence due to the presence of multiple local minima. In this survey the principal methods are explained in detail. Furthermore, the most important ones are simulated in order to determine which method is better depending on the conditions of the problem. In order to compare them, experimental results are realized using synthetic and real data. The quality of some results indicates 


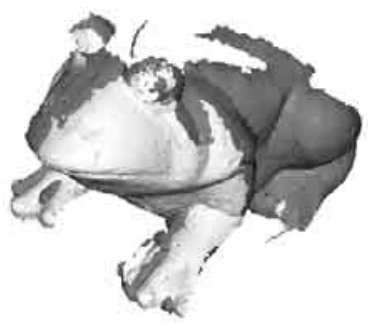

(a) Besl

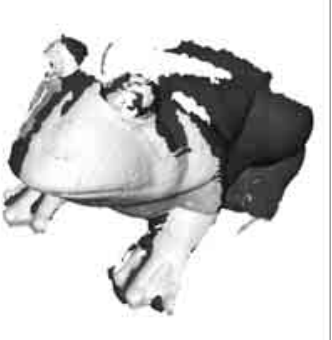

(b) Jost

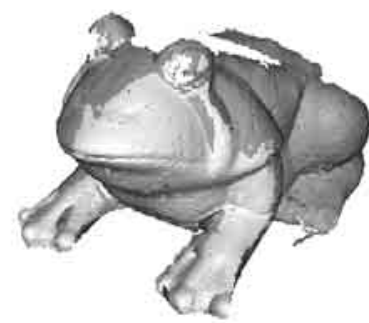

(c) Trucco

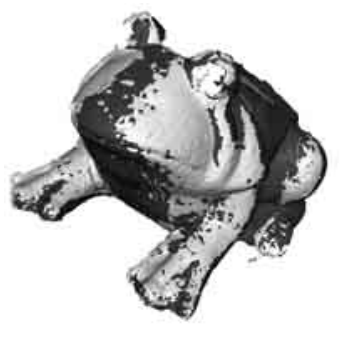

(d) Chow

Figure 2. Exemples of final Pair-wise Registration

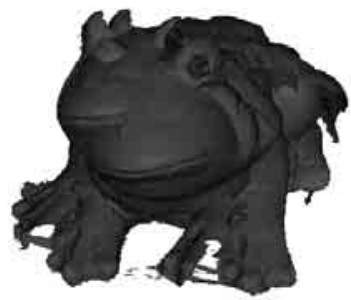

(a) Traditional Pair-wise

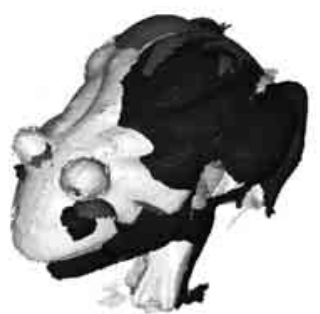

(b) Our approach without bad views correction

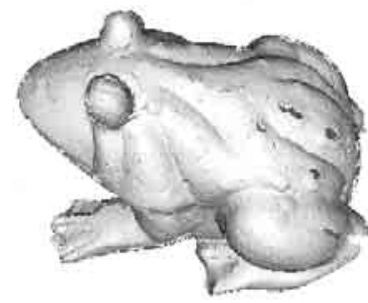

(c) Final Registration

Figure 3. Full Reconstruction

that the result of the registration can be used to compare a real object with its $3 \mathrm{D}$ models. It can be used in manufacturing process enabling the inspection of the quality of the produced objects.

Analyzing the results of Coarse registration methods, we thought that for images of low resolution the RANSAC-based method of Chen is the best. In spite of, the accuracy of this method increments with the number of points used, the required time to find a solution is a problem to solve. When more points are used, the best method is the Spin Image, however, they require a lot of time, too. In this situation, and if the time is an important factor, the Principal Component Analysis is the best method. The problem of this method is that only aligns the three principal axis, and the solution of the method can be an error of 90 or 180 . This error can be solved by modifying the problem in order to find the solution that maximize the number of overlapping points.

The method of Chen is useful to search a good initial solution, however it presents the problem of the computing time. The method of PCA is the fastest, but requires an important overlapping region and have the problem of symmetries and a possible solution displaced 90 or 180 . The Spin Image is a good method to obtain and initial estimation, especially if the face-based spin image is used. Genetic algorithms are robust to noise and provide good results, however, the method requires a too high computing time.

¿From our point of view, the method of ICP is the best of the Fine registration methods. In spite of the results of the proposal of Besl are not very good, this fact is due to that the motion is computed using points that do not belong to the overlapping region. To solve this, it is necessary to use a robust method, like Zim $\beta$ er of Trucco. The difference between them is how to they estimate the standard deviation in each iteration. Although both strategies are good, the results indicate that the method of Zimßer is more accurate than Trucco, on the other hand, the method of Trucco is faster when the number of points is high. The variant of Jost presents good results and the increment of the consuming time is very important. A combination of it with a robust method is probably the best solution to register range images. The results indicate that Chen's method is more sensitive to the problem of local minima, this fact decreases the quality of the results. The GAs present good results, however the required time is much larger than the others. Furthermore, this time increases when the solution is far from the real one. 
Moreover, the combination of Pair-wise registration algorithms with our proposal lets us to obtain full reconstructions with good accuracy. This final model can be used to evaluate the quality of manufactured products comparing this model with the initial requirement. Our method can be applied to all objects, because the control is neither destructive nor aggressive.

\section{ACKNOWLEDGMENTS}

Work founded by Spanish Project TIC2003-08106-C02-02.

\section{REFERENCES}

1. J. Forest and J. Salvi, "An overview of laser slit 3d digitasers," in International Conference on Robots and Systems, pp. 73-78, (Lausanne), October 2002.

2. J. Salvi, J. Pagès, and J. Batlle, "Pattern codification strategies in structured light systems," Pattern Recognition 37, pp. 827-849, April 2004.

3. J. Salvi, X. Armangué, and J. Batlle, "A comparative review of camera calibrating methods with accuracy evaluation," Pattern Recognition 35, pp. 1617-1635, July 2002.

4. C. J. R. Chua, "Point signatures: A new representation for 3d object recognition," International Journal of Computer Vision 25, pp. 63-85, October 1997.

5. A. Johnson, "Spin-images: A representation for 3-d surface matching.," in Ph. D., 1997.

6. O. Carmichael, D. Huber, and M. Hebert, "Large data sets and confusing scenes in 3-d surface matching and recognition," in Second International Conference on 3-D Digital Imaging and Modeling, pp. 258-367, (Ottawa, Ont. , Canada), October 1999.

7. D. Huber and M. Hebert, "A new approach to 3-d terrain mapping," in International Conference on Intelligent Robotics and Systems, pp. 1121-1127, October 1999.

8. D. Chung, Y. D., and S. Lee, "Registration of multiple-range views using the reverse-calibration technique," Pattern Recognition 31(4), pp. 457-464, 1998.

9. C.-S. Chen, Y.-P. Hung, and J.-B. Cheng, "A fast automatic method for registration of partially overlapping range images," in International Conference on Computer Vision, pp. 242-248, (Bombay), January 1998.

10. K. Brunnström and A. Stoddart, "Genetic algorithms for free-form surface matching," in International Conference of Pattern Recognition, pp. 689-693, (Vienna), 1996.

11. P. Besl and N. McKay, "A method for registration of 3-d shapes," PAMI 14, pp. 239-256, February 1992.

12. E. Trucco, A. Fusiello, and V. Roberto, "Robust motion and correspondences of noisy 3-d point sets with missing data," Pattern Recognition Letters 20, pp. 889-898, September 1999.

13. P. Rousseeuw and A. Leroy, Robust Regression and Outlier Detection, Wiley, New York, 1987.

14. T. Zimßer and H. Schnidt, J. Niermann, "A refined icp algorithm for robust 3-d correspondences estimation," in International Conference on Image Processing, pp. 695-698, September 2003.

15. T. Jost and H. Hugli, "A multi-resolution scheme icp algorithm for fast shape registration," in First International Symposium on 3D Data Processing Visualization and Transmission, pp. 540- 543, 2002.

16. G. Chen, Y. ad Medioni, "Object modeling by registration of multiple range images," in IEEE International Conference on Robotics and Automation, pp. 2724 -2729, April 1991.

17. H. Gagnon, M. Soucy, R. Bergevin, and D. Laurendeau, "Registration of multiple range views for automatic 3-d model building," Computer Vision and Pattern Recognition, pp. 581 -586, June 1994.

18. T. Masuda, "Generation of geometric model by registration and integration of multiple range images," in Third International Conference on 3-D Digital Imaging and Modeling, pp. 254-261, May 2001.

19. C. Chow, H. Tsui, and T. Lee, "Surface registration using a dynamic genetic allgorithm," Pattern recognition 37, pp. 105-117, January 2004. 